\title{
Non-Homogeneous Uncoupled Beam under Axially Distributed Loads
}

\author{
M. Grebshtein, M. Kazar (Kezerashvili), V. Rovenski and O. Rand \\ Faculty of Aerospace Engineering, Technion - Israel Institute of Technology, Israel
}

\begin{abstract}
The paper presents a derivation for the analysis of monoclinic beams of non-homogeneous cross-section that undergo axially distributed surface loads and body forces. The derivation has been proved to be exact by symbolic computational tools, and includes illustrative numerical examples.
\end{abstract}

Keywords: Non-homogeneous monoclinic beam, Neumann problem, biharmonic problem, auxiliary problems of plane deformation.

\section{INTRODUCTION}

The paper presents the study of anisotropic beams with a non-homogeneous cross-section, that consists of various $Z$-monoclinic materials, where the $(x-y)$ planes of the cross-sections coincide with the planes of elastic symmetry.

The origin of the method developed in this paper seems to be Michell /1/, where the problem solution for homogeneous isotropic beam under surface loads that do not vary along the generators is expressed by three harmonic and one biharmonic functions. Independently Almansi $/ 2 /$, presented an analytical (level-based) solution for the same problem when the surface loads are polynomials of the beam axis variable. Hence, many of the related works are generally referred to as "Michell-Almansi (recursive) method". An analogous methodology, founded on prescribed stress (and not deformation) distributions, has been presented much later by Dzhanelidze /3/. Similar problems were also discussed by Love /4/, Muskhelishvili /5/,
Kosmodamianskii /6/ and others. The above solutions were further evolved by Ruchadze /7/ for homogeneous isotropic beams undergoing both surface and body loads. The formulation was first valid for loads that may be longitudinally expressed by third order polynomials only. Later on, Ruchadze and Berekashvili $/ 8 /$ and Zivsivadse and Bereksashvili /9/ extended the method for the case of Z-monoclinic beams and generic polynomial loading.

This paper presents an improved derivation of the above approach. We use notations from $/ 10 /$ for nonhomogeneous domain, interlaminar and boundary conditions, generic Neumann-type problems and the auxiliary problems of plane deformation.

To prove the symbolic exactness of the expressions, the entire methodology is documented and verified symbolically by Maple. The illustrative examples are also produced by Maple.

\section{THE SOLUTION HYPOTHESIS AND PROCEDURE}

The constitutive relations for Z-monoclinic material are given by the linear Hook's law $\left[\varepsilon_{x}, \varepsilon_{y}, \varepsilon_{z}, \gamma_{y z}, \gamma_{x z}, \gamma_{x y}\right]=a\left[\sigma_{x}, \sigma_{y}, \sigma_{z}, \gamma_{y z}, \gamma_{x z}, \gamma_{y y}\right]$ with a positive definite matrix $a$ (with 13 independent coefficients)

$$
a=\left[\begin{array}{cccccc}
a_{11} & a_{12} & a_{13} & 0 & 0 & a_{16} \\
& a_{22} & a_{23} & 0 & 0 & a_{26} \\
& & a_{23} & 0 & 0 & a_{36} \\
& & & a_{44} & a_{45} & 0 \\
& \text { Sym. } & & & a_{55} & 0 \\
& & & & & a_{66}
\end{array}\right]
$$


Such typical materials may be obtained by rotating orthotropic material about the beam $(z)$ axis. We assume the most general surface loading form (per unit area), $\mathbf{F}_{s}=\left\{X_{s}, Y_{s}, Z_{s}\right\}$, and distributed body forces (per unit volume), $\mathbf{F}_{b}=\left\{X_{b}, Y_{b}, Z_{b}\right\}$, see Figure 1 , which are expressed as vector polynomials of degree $K>0$,

$$
\begin{aligned}
& \mathbf{F}_{s}=\sum_{k=0}^{K}\left\{X_{s}^{(k)}, Y_{s}^{(k)}, Z_{s}^{(k)}\right\}(x, y) z^{k}, \\
& \mathbf{F}_{b}=\sum_{k=0}^{K}\left\{X_{b}^{(k)}, Y_{b}^{(k)}, Z_{b}^{(k)}\right\}(x, y) z^{k} .
\end{aligned}
$$

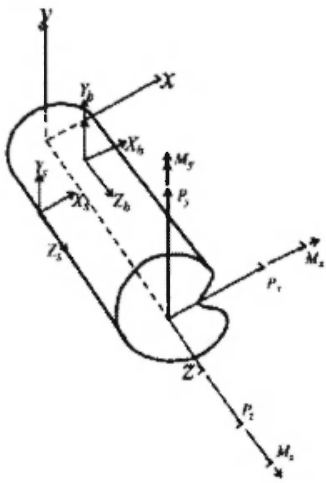

a) General view.

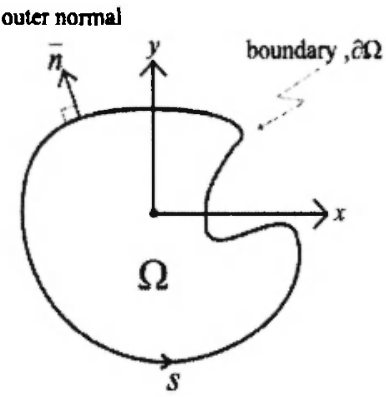

b) A cross-section.
Fig. 1: Beam.

Hence, the case of $K=0$ stands for uniform distributed loads in the $z$-direction, $K=1$ stands for linear distribution, etc. In general, we let each of the two stress components $\tau_{x z}, \tau_{y z}$ to be polynomials of degree $K+1$ in $z$, while $\sigma_{x}, \sigma_{y}, \tau_{x y}$ and $\sigma_{z}$ are polynomials of degree $K+2$ in $z$ as

$$
\begin{aligned}
& \left\{\bar{\sigma}_{x}, \bar{\sigma}_{y}, \bar{\tau}_{x y}, \bar{\sigma}_{z}\right\}=\sum_{k=0}^{K+2}\left(\bar{\sigma}_{x}^{(k)}, \bar{v}_{y}^{(k)}, \bar{\tau}_{x y}^{(k)}, \bar{\sigma}_{z}^{(k)}\right\} z^{k}, \\
& \left\{\tau_{z z}, \tau_{y z}\right\}-\sum_{k=0}^{K+1}\left\{\bar{\tau}_{x z}^{(k)}, \tau_{y z}^{(k)}\right\} z^{k},
\end{aligned}
$$

where $\sigma_{i}^{(k)}, \tau_{i j}^{(k)}$ are functions of $x, y$. In the homogeneous case, we let each of the three stress components $\sigma_{x}, \sigma_{y}, \tau_{x y}$ be expressed as polynomials of degree $K$ in $z$.
Table 1

Solution procedure for axially non-uniform loading of a Z-monoclinic beam.

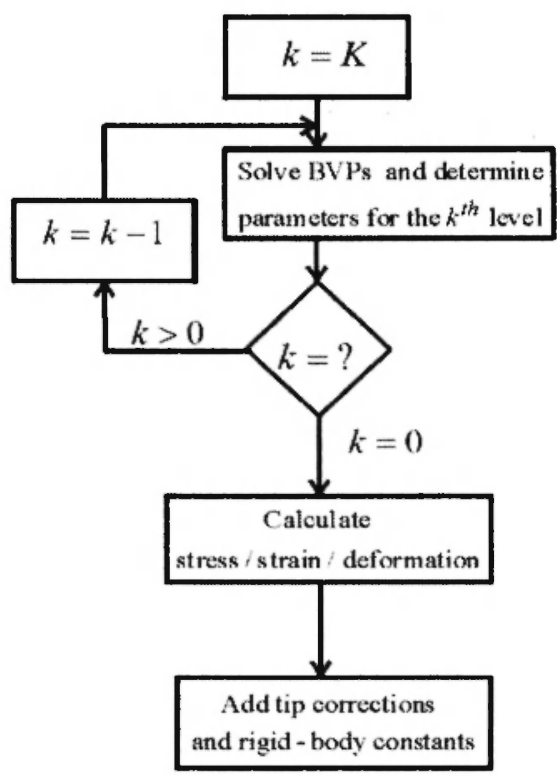

A scheme of the level-based solution methodology is presented in Table 1. The process is initiated for $k=K$ and continues for lower levels down to $k=0$. For each $K$ $\geq k \geq 0$ level, a set of the biharmonic and the Neumann problems in non-homogeneous domain $\Omega$ should be solved. Each solution level is driven by its level loading, and the quantities obtained in previous (higher) levels. As shown by Table 2, the solution ingredients are gradually introduced according to their level of appearance.

It should be noted that the solution expressions are "symmetric" under the following parameter interchange: $x \leftrightarrow y, 1 \leftrightarrow 2,4 \leftrightarrow 5, X_{b} \leftrightarrow Y_{b}, X_{s} \leftrightarrow Y_{s}$, etc., see Table 2. One may obtain symmetric expressions using operation "Sym". For example, $u \leftrightarrow v$ means $u=\operatorname{Sym}(v)$ and $v=\operatorname{Sym}(u)$. Also, $Q^{\chi_{1}} \leftrightarrow P^{\chi_{2}}, Q^{\chi_{2}} \leftrightarrow P^{\chi_{1}}, Q^{\varphi} \leftrightarrow-P^{\varphi}, D_{2}=-\operatorname{Sym}\left(D_{1}\right)$ and $\tau=-\operatorname{Sym}(\tau), \sigma_{z}=\operatorname{Sym}\left(\sigma_{z}\right)$. This solution does not ensure that the three forces and three moments at the beam tip vanish, see stress integral

$$
\begin{aligned}
& \iint_{\Omega}\left\{\sigma_{z}, \sigma_{z} y, \sigma_{z} x, \tau_{x z}, \tau_{x z}, \tau_{y z} x-\tau_{x z} x\right\}= \\
& \quad\left\{P_{z}, M_{x},-M_{y}, P_{x}, P_{y}, M_{z}\right\} .
\end{aligned}
$$


Table 2

Maximal level of appearance and symmetry of various solution components.

\begin{tabular}{|c|c|}
\hline Component & Level \\
\hline$\sigma_{x} \leftrightarrow \sigma_{y}, \tau_{x y} \leftrightarrow \tau_{x y}$ & $K+2$ \\
\hline$\tau_{x z} \leftrightarrow \tau_{y z}, \gamma_{x z} \leftrightarrow \gamma_{y z}$ & $K+1$ \\
\hline$\sigma_{z} \leftrightarrow \sigma_{z}, \varepsilon_{z} \leftrightarrow \varepsilon_{z}$, & $K+2$ \\
$\varepsilon_{x} \leftrightarrow \varepsilon_{y}, \gamma_{x y} \leftrightarrow \gamma_{x y}$ & \\
\hline$u \leftrightarrow v$ & $K+4$ \\
\hline$w \leftrightarrow w$ & $K+3$ \\
\hline$\omega_{x} \leftrightarrow-\omega_{y}$ & $K+3$ \\
\hline$\omega_{z} \leftrightarrow-\omega_{z}$ & $K+2$ \\
\hline$H, S_{x} \leftrightarrow S_{y}$ & $K+1$ \\
\hline$p \leftrightarrow q, \tau \leftrightarrow-\tau, d \leftrightarrow d$ & $K$ \\
\hline$\Phi, \omega, L \leftrightarrow M$ & $K$ \\
\hline$X_{b}, Y_{b}, Z_{b}, X_{s}, Y_{s}, Z_{s}, \bar{U}_{1} \leftrightarrow \bar{U}_{2}$ & $K$ \\
\hline$Q^{\omega} \leftrightarrow P^{\omega}, Q_{2} \leftrightarrow A, Q_{1} \leftrightarrow P_{2}$ & $K$ \\
\hline
\end{tabular}

Hence, one needs to superimpose a series of solutions for tip loads (see $/ 10 /$ ) in order to cancel out these resultants.

\subsection{Stress components}

The detailed stress expressions are

$$
\begin{aligned}
& \sigma_{x}=\sum_{k=0}^{K} z^{k}\left(\Phi_{, y y}^{(k)}+\bar{U}_{:}^{(k)}-\frac{k+1}{(4)}\left[a_{44} H^{(k+1)}\right.\right. \\
& -\frac{d_{k+1} a_{0}}{4 a_{33}}\left(\frac{3 a_{23}+a_{44}}{a_{13}} y^{2}-x^{2}\right)+S_{:}^{(k+1)} \mid \\
& \left.-\frac{z}{k+1} d_{k} \bar{\sigma}_{y}^{(3)}+\frac{z^{2}}{(k+1)(k+2)}\left(p_{k} \bar{\sigma}_{x}^{(1)}+q_{k} \bar{\sigma}_{x}^{(2)}\right)\right\}, \\
& \sigma_{z}=\sum_{k=0}^{K} \frac{z^{k}}{a_{33}}\left\{-a_{13}\left(\Phi_{. v v}^{\left(k^{2}\right.}+\bar{U}_{1}^{(k)}\right)-a_{23}\left(\Phi_{. x x}^{\prime \prime)}+\bar{U}_{2}^{(k)}\right)\right. \\
& \left.+a_{36} \Phi_{, x y}^{(k)}+i p l v+a_{l} x\right) x v+\frac{k+1}{\left.a_{0}\right)}-\mid\left(a_{13} a_{44}+a_{23} a_{55}\right. \\
& \left.-a_{36} a_{45}+a_{0}\right) l^{(k+1)}-\frac{d_{k+1} a_{0}}{2 a_{33}}\left(a_{45}+a_{36}\right) x y \\
& +a_{13} s_{x}^{(k+1)}+a_{23} s_{y}^{(k+1)} \mid-\frac{z d_{k}}{k+1}\left(1+a_{33} \bar{\sigma}_{z}^{(3)}\right) \\
& \left.\left.-\frac{z^{2}}{(k+1)(k+2)} \mid p_{k}\left(x-a_{33} \bar{\sigma}_{z}^{(1)}\right)+q_{k}\left(y-a_{33} \bar{\sigma}_{z}^{(2)}\right)\right]\right\}, \\
& \tau_{y z}=\sum_{k=0}^{k+1} \frac{\bar{z}^{k}}{a_{0}}\left(a_{55} H_{, y}^{(k)}-a_{45} H_{. x}^{(k)}+\frac{d_{k} a_{0}}{2 a_{33}} y+S_{y, y}^{(k)}\right),
\end{aligned}
$$

$$
\begin{aligned}
& \tau_{x y}=\sum_{k=0}^{K} z^{k}\left[-\Phi_{x y}^{(k)}+(k+1) \frac{a_{45}}{a_{n}} H^{(k+1)}-\frac{z d_{k}}{k+1} \bar{\tau}_{x y}^{(3)}\right. \\
& \left.+\frac{\epsilon^{2}}{(k+1)(k+2)}\left(p_{k} \bar{z}_{x y}^{(1)}+q_{k} \tau_{x y}^{\prime 2)}\right)\right], \\
& \sigma_{y}=\operatorname{Sym}\left(\sigma_{y}\right), \quad \tau_{x z}=\operatorname{Sym}\left(\tau_{y z}\right) .
\end{aligned}
$$

The body force potentials $\bar{U}_{j}^{(k)}(k=0, \ldots, K)$ that appear in the above terms are

$$
\bar{U}_{1}=-\int_{b}^{x} X_{b} d x=\sum_{k=0}^{K} \bar{U}_{1}^{(k)}(x, y) z^{k}, \bar{U}_{2}=\operatorname{Sym}\left(\bar{U}_{1}\right)
$$

The expressions for $S_{x}^{(i) i}, S_{v}^{(i, j)}(k=0, \ldots, K+1)$ are

$$
\begin{aligned}
& S_{x}^{(k)}=\int_{0}^{x}\left[(k+1)\left(a_{44} L^{(k+1)}-a_{45} M^{(k+1)}\right)\right. \\
& -\tilde{\delta}_{k 0}\left(p_{k-1} p^{\chi_{1}}+q_{k-1} p^{\chi_{2}}-\tau_{k-1} p^{\varphi}\right) \\
& \left.-d_{k}\left(a_{44} \bar{u}^{(3)}-a_{45} \bar{v}^{(3)}\right)\right] d x \\
& S_{y}^{(k)}=\operatorname{Sym}\left(S_{x}^{(k)}\right)
\end{aligned}
$$

where $\tilde{\delta}_{k 0}=1 / k$ for $k \neq 0$ and $\tilde{\delta}_{00}=0$, the polynomials $P^{\chi k}, Q^{\chi k}, P^{\varphi}, Q^{\varphi}$ are defined in $/ 10 /$. In (5) and in all expressions below, we replace $H^{(k)}(k<K+1)$ by the RHS of

$$
I^{(k)}=\omega^{(k)}+\bar{\delta}_{k 0}\left(p_{k-1} \chi_{1}+q_{k-1} \chi_{2}-\tau_{k-1} \varphi\right)
$$

where $\omega^{(k)}(k<K)$ is an additional series of longitudinal stress functions as required by the singlevalue conditions for the present problem, see item (e) in Section 2.

Remark 1. The problem without single-valued requirements for the biharmonic stress function may be considered. In this case one may assume $p_{k}=q_{k}=\bar{r}_{n}=0$ and use the harmonic stress functions $H^{(k)}$ only, i.e., without definition (8).

\subsection{Displacements}

The strain components are derived from (5), using Hook's law, see (1). Displacements are determined via 
integration of strains. The rigid-body displacements are not included in these expressions and are introduced by the tip loads correction, see Section 1:

$$
\begin{gathered}
u=\sum_{k=0}^{K} z^{k} ! L^{(k)} \frac{z d_{k}}{k+1}\left(\frac{2 a_{13} x+a_{36} y}{2 a_{33}}+\bar{u}^{(3)}\right) \\
-\frac{z^{4} p_{k}}{(k+1)(k+2)(k+3)(k+4)} \\
-\frac{z^{2}}{(k+1)(k+2)}\left[n_{n}\left(\frac{a_{23} y^{2}-a_{13} x^{2}}{2 a_{33}}-\bar{u}^{(1)}\right)\right. \\
\left.\left.-q_{k}\left(\frac{a_{36} v^{2}+2 a_{13} x v}{2\left(a_{33}\right.}+\bar{u}^{(2)}\right)-y \tau_{k}\right]\right\}, \\
v=S y m(u), \\
w^{\prime}=\sum_{k=0}^{K+1} z^{k}: H^{(k)}+\frac{d_{k}}{4 a_{33}}\left[\left(2 a_{13}+a_{55}\right) x^{2}\right. \\
+2\left(a_{36}+a_{45}\right) x v+\left(2 a_{23}+a_{44}\right) v^{2} \mid-\frac{z^{2} d_{k}}{(k+1)(k+2)} \\
\left.-\frac{z}{k+1}\left(q_{k} x+p_{k} v\right) x v+\frac{z^{3}\left(n_{n}^{\prime} x+g_{n} y\right)}{(\dot{k}+i k \dot{k}+2)(\dot{k}+3)}\right\} .
\end{gathered}
$$

\subsection{Biharmonic stress functions}

To enable further handling of the boundary conditions, we shall also use the following general identities for $C^{2}$-differentiable functions:

$$
\begin{aligned}
& \frac{d}{d s} \Phi_{y}^{(k)}=\Phi_{u}^{(k)} \cos (n, x)-\Phi_{, y}^{(k)} \cos (n, y) . \\
& \frac{d}{d s} \Phi_{. r}^{(k)}=\Phi_{, y}^{(k)} \cos (n, x)-\Phi_{y x}^{(k)} \cos (\dot{n}, y) .
\end{aligned}
$$

The biharmonic stress functions $\Phi^{(k)}(k=0, \ldots, K)$ are governed by

$$
\begin{aligned}
& \nabla_{1}^{(4)} \Phi^{(k)}=F_{0}^{(k)} \text { over } \Omega, \\
& \frac{d}{d s}\left\{\Phi_{, *}^{(k)}, \Phi_{.,}^{(k)}\right\}=\left\{-F_{1}^{(k)}, F_{2}^{(k)}\right\} \text { in } \partial \Omega, \\
& \frac{d}{d s}\left\{\Phi_{x}^{(k)}, \Phi_{y}^{(k)}\right\}_{[i]}^{[j]}=\left\{-F_{1}^{(k)}, F_{2}^{(k)}\right\}_{[i]}[j] \text { in } \partial \Omega_{i j}, \\
& \left\{L^{(k)}, M^{(k)}\right\}[j]=\{0,0\} \text { in } \partial \Omega_{i j}
\end{aligned}
$$

where $F_{i}^{k}=P_{i}^{k} \cos (\dot{n}, x)+Q_{i}^{k} \cos (\dot{n}, y)$ and

$$
\begin{aligned}
& F_{0}^{(k)}=-\left(b_{1} 1 U_{i}^{(k)}+b_{12} U_{2}^{(i k)}\right) \\
& +\left(b_{16} U_{1}^{(k)}+b_{26} \bar{U}_{2}^{(k)}\right)_{1 . x}-\left(b_{12} \bar{U}_{1}^{(k)}+b_{22} \bar{U}_{2}^{(k)}\right)_{x x} \\
& -\frac{2 b_{L}}{a_{33}}\left(a_{13} x-a_{36} v\right)+\frac{2 a_{L}}{a_{33}}\left(a_{36} x-a_{23} v\right) \\
& +(k+1)\left\{\left(\frac{0_{11} a_{44}+b_{12} a_{55}-b_{16} a_{45}}{a_{0}}-\frac{a_{13}}{a_{33}}\right) H_{33}^{(k+1)}\right. \\
& +\left(\frac{b_{12} a_{44}+b_{22} a_{55}-b_{26} a_{45}}{a_{0}}-\frac{a_{23}}{a_{33}}\right) H_{\ldots . r}^{(k+1)} \\
& -\left(\frac{b_{16} a_{44}+b_{26} a_{55}-b_{66} a_{45}}{a_{0}}-\frac{a_{36}}{a_{33}} ; i i_{(5)}^{(k+1)}\right. \\
& \left.+\frac{d_{t+1}}{2 a_{33}} i z a_{1}\right)+\frac{a_{36}}{a_{33}}\left(a_{36}+a_{45}\right)-\frac{a_{11}}{a_{13}}\left(3 a_{23}+a_{44}\right) \\
& -\frac{a_{22}}{a_{23}}\left(3 a_{13}+a_{55}\right) 1+\frac{1}{a_{0}} \mid\left(b_{11} S_{r}^{(k+1)}+b_{12} S_{1}^{(k+1)}\right)_{1,1} \\
& \left.\left.+\left(b_{12} S_{x}^{(k+1)}+b_{22} S_{y}^{(k+1)}\right)_{, x x}+\left(b_{16} S_{x}^{(k+1)}+b_{26} S_{y}^{(k+1)}\right)_{, y y}\right]\right\} \text {. } \\
& P_{1}^{(k)}=\gamma_{1}^{(k)}-(k+1) \frac{a_{45}}{a_{0}} H^{(k+1)} \text {. } \\
& Q_{1}^{(k)}=Y_{2}^{(k)}-\bar{U}_{2}^{(k)}+\frac{k+1}{a_{0}} \mid a_{55} H^{(k+1)} \\
& -\frac{d_{k+1} a_{0}}{4 a_{33}}\left(\frac{3 a_{13}+a_{55}}{a_{23}} r^{2}-v^{2}\right)+S_{y}^{(k+1)} \mid . \\
& Q_{2}^{(k)}=\operatorname{Sym}\left(P_{1}^{(k)}\right), P_{2}^{(k)}=\operatorname{Sym}\left(Q_{1}^{(k)}\right)
\end{aligned}
$$

The biharmonic operator of $(10 a)$ is defined as

$$
\begin{aligned}
& \nabla_{1}^{(4)}=b_{22} \frac{\partial^{4}}{\partial x^{4}}-2 b_{26} \frac{\partial^{4}}{\partial x^{3} c^{3}} \\
& +\left(2 b_{12}+b_{66}\right) \frac{\partial^{4}}{\partial x^{2} \partial y^{2}}-2 m_{16} \frac{\partial^{4}}{\partial x \partial^{3}}+b_{11} \frac{\partial^{4}}{\partial y^{4}} .
\end{aligned}
$$

The ellipticity of $\nabla_{1}^{(4)}$ follows from positive definite Hook's law. (1), see $/ 12 /$. The $\partial / \partial s$-type boundary conditions (10b,c) leave the values of the functions $\Phi^{(k)}$ and the derivatives $\Phi_{x}^{(k)}, \Phi_{* i}^{(i)}$ undetermined by a constant for each homogeneous domain component. As a general rule we select a point over each dividing curve, (say, $\left.\left(x_{i}^{\eta}, y_{1}^{i \prime}\right) \in i \Omega_{i j}\right)$, where we force the functions to be equal for both neighbor domains. namely, $\left\{\Phi^{(k)}, \Phi_{x}^{(k)}, \Phi_{, y}^{(k)}\right\}\left(x_{1}^{i}, y_{i}^{i j}\right)_{|i|}^{|j|}=\{0,0.0\}$.

For the sake of simplicity we also assume

$$
\left\{\Phi^{(k)} \cdot \Phi_{x}^{(k)} \cdot \Phi_{\cdot}^{(k)}\right\}(0,0)=\{0,0,0\} .
$$


The single-valued conditions for biharmonic function $\Phi^{(k)}$ on a simply connected (homogeneous) domain are, see $/ 11 /$,

$$
\oint_{\partial \Omega}\left\{F_{1}^{(k)}, F_{2}^{(k)}, F_{2}^{(k)} y-F_{1}^{(k)} x\right\}=\{0,0,0\}
$$

Subsequently, the single-valued type conditions, necessary for solution of (10) existence on a nonhomogeneous domain are

$$
\begin{aligned}
& \oint_{a \Omega}\left\{F_{1}^{(k)}, F_{2}^{(k)}, F_{2}^{(k)} y-F_{1}^{(k)} x\right\} \\
& +\sum_{i j} \oint_{\Omega_{i j}}\left\{F_{1}^{(k)}, F_{2}^{(k)}, F_{2}^{(k)} y-F_{1}^{(k)} x\right\}_{[i]}^{[j]}=\{0,0,0\} .
\end{aligned}
$$

These equalities become clearer if (13) are written first for each domain component $\Omega_{[J]}$ and then summed up.

\subsection{Longitudinal stress functions}

The Laplace-type operator and Neumann-type boundary operator are, see /10/,

$$
\begin{aligned}
& \nabla_{3}^{(2)}=a_{44} \frac{\partial^{2}}{\partial x^{2}}-a_{4} \xi \frac{\partial^{2}}{\partial x \partial y}+a_{55} \frac{\partial^{2}}{\partial y^{2}} \\
& D_{i}^{n}=\left(a_{44} \frac{\partial}{\partial x}-a_{45} \frac{\partial}{\partial y}\right) \cos (\vec{n}, x) \\
& \quad+\left(-a_{45} \frac{\partial}{\partial x}+a_{55} \frac{\partial}{\partial y}\right) \cos (\vec{n}, v) .
\end{aligned}
$$

The longitudinal stress functions $\omega^{(k)}(k=0, \ldots, K)$ are governed by the Neumann problem

$$
\begin{aligned}
& \nabla_{j}^{(2)} \omega^{(k)}=F_{0}^{\left(\omega^{(k)}\right.} \text { over } \Omega, \\
& D_{1}^{n} \omega^{(k)}=F_{3}^{(k)} \text { on } \partial \Omega, \\
& {\left[\frac{1}{a_{0}}\left(D_{1}^{n} \omega^{(k)}-F_{3}^{(k)}\right)\right]_{[i]}^{[j]}=0 \text { on } \partial \Omega_{i j},} \\
& {\left[\omega^{(k)}\right]_{[i,}^{[j]}=F^{\omega^{(k)}, i j} \text { on } \partial \Omega_{i j}}
\end{aligned}
$$

where $F_{3}^{(k)}=P^{\omega^{(k)}} \cos (\vec{n}, x)+Q^{\omega^{(n)}} \cos (\vec{n}, y)$ and

$$
\begin{aligned}
& P^{\left(\omega^{(k)}\right.}=a_{0} Z_{1}^{(k)}+d_{k}\left(-\frac{a_{0}}{2 a_{33}} x+a_{44} \bar{u}^{(3)}-a_{45} \bar{v}^{(3)}\right) \\
& -(k+1)\left(a_{44} L^{(k+1)}-a_{45} M^{(k+1)}\right), \\
& Q^{\omega^{(k)}}=\operatorname{Sym}\left(P^{\omega^{(k)}}\right) \text {, }
\end{aligned}
$$

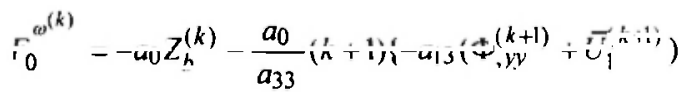

$$
\begin{aligned}
& -a_{23}\left(\Phi_{, x x}^{(k+1)}+\bar{U}_{2}^{(k+1)}\right)+a_{36} \Phi_{x y}^{(k+1)}+\left(p_{k+1} y+q_{k+1} x\right) x y \\
& -(k+1)\left(a_{44} L_{, x}^{(k+1)}+a_{55} M_{, y}^{(k+1)}-a_{45} L_{y}^{(k+1)}-a_{45} M_{, x}^{(k+1)}\right) \\
& +(k+2)\left[\frac{a_{13} a_{44}+a_{23} a_{55}-a_{36} a_{45}+a_{0}}{a_{0}} H^{(k+2)}\right. \\
& \left.+\frac{d_{k+2}}{2 a_{33}^{?}}\left(a_{45}+a_{36}\right) x y+\frac{a_{13}}{a_{0}} S_{x}^{(k+2)}+\frac{a_{23}}{a_{0}} S_{y}^{(k+2)} \mid\right\} \\
& +d_{k}\left(a_{45} \vec{\varepsilon}_{x}^{(3)}+a_{55} \vec{\varepsilon}_{y}^{(3)}-a_{45} \vec{\gamma}_{x y}^{(3)}+a_{0} \sigma_{z}^{(3)}\right) \text {. } \\
& F^{\left.\omega^{(k)}, i\right)}=-d_{k}\left\{\frac { 1 } { 4 a _ { 3 3 } } \left[\left(2 a_{13}+a_{55}\right) x^{2}+2\left(a_{36}+a_{45}\right) x y\right.\right. \\
& \left.\left.\left.+\left(2 a_{23}+a_{44}\right) y^{2}\right]\right\}\right\}_{\{,}^{[1]} \text {. }
\end{aligned}
$$

For convenience we assume $\omega^{(k)}(0,0)=0$. The necessary condition for the existence of a solution to boundary value problem (16), see $/ 10 /$, is

$$
\oint_{\partial \Omega} \frac{1}{a_{0}} F_{3}^{(k)}+\sum_{y} \int_{\Omega_{i j}}\left[\frac{1}{a_{0}} F_{3}^{(k)}\right]_{[i]}^{[j]}=\iint_{\Omega} \frac{1}{a_{0}} F_{0}^{\left(\omega^{(k)}\right.} .
$$

In contrast to torsion and bending stress functions, $\omega^{(k)}$ may be discontinuous along dividing contours $\Omega_{i j}$, see (16d).

\subsection{Auxiliary functions}

The functions $L^{(k)}(x, y)(k=0, \ldots, K)$ are defined by recurrence formula

$$
\begin{aligned}
& L^{(k)}=b_{12} \Phi_{, x}^{(k)}-b_{16} \Phi_{, y}^{(k)}+\prod_{j}^{k x} ! b_{11}\left(\Phi_{y}^{\prime \prime \prime}+\bar{U}_{1}^{(k)}\right)+b_{12} \bar{U}_{2}^{(k)} \\
& -(k+1)\left[\left(\frac{b_{11} a_{44}+b_{12} a_{55}-b_{16} a_{45}}{a_{0}}-\frac{a_{13}}{a_{33}}-\right) H^{(k+1)}+\frac{b_{11}}{a_{0}} S_{\mathrm{x}}^{(k+1)}\right. \\
& \left.\left.+\frac{b_{12}}{a_{0}} S_{y}^{(k+1)}\right]\right\} d x+\frac{p_{k}}{a_{33}}\left\{1 \frac { 1 } { a _ { 0 } } \left(\left(b_{26} a_{45}+b_{12} a_{44}\right)\left(a_{23}+2 a_{33}\right)\right.\right. \\
& \left.\left.\left.+a_{55} b_{22}\left(2 a_{33}-a_{23}\right)\right)-2 a_{23}\right] \frac{y^{4}}{24}+a_{13} \frac{x^{2} y^{2}}{2}\right\}+\frac{a_{t}}{a_{33}}\left\{a_{13} \frac{x^{3} y}{3}\right. \\
& -\frac{1}{a_{0}}\left[a_{45} b_{22}\left(2 a_{33}-a_{13}\right)+b_{12}\left(a_{36} a_{44}-a_{23} a_{45}\right)-b_{26}\left(a_{23} a_{55}\right.\right. \\
& \left.\left.\left.-a_{36} a_{45}\right)\right] \frac{y^{4}}{24}\right\}+\frac{\tau t}{a_{0}}\left(b_{12} a_{44}-b_{22} a_{55}+b_{26} a_{45}\right) \frac{y^{3}}{\mathrm{D}}
\end{aligned}
$$




$$
\begin{aligned}
& +(k+1) \frac{d_{k+1}}{4 a_{33}}\left\{\left[\frac{a_{12}\left(3 a_{13}+a_{55}\right)}{a_{23}}-0: 1: \frac{x^{3}}{3}\right.\right. \\
& +\frac{a_{13}\left(a_{45}+a_{36}\right)}{a_{33}} x^{2} y+\left[\frac{a_{11}\left(3 a_{23}+a_{44}\right)}{a_{13}}-a_{12}\right] x y^{2} \\
& \left.-\left[a_{26}+\frac{a_{23}\left(a_{45}+a_{36}\right)}{a_{33}}-\frac{a_{16}\left(3 a_{23}+a_{44}\right)}{a_{13}}\right] \frac{y^{3}}{3}\right\}-\int_{0}^{y} l^{(k)}(y) d y, \\
& l^{(k)}(y)=\left\{\int_{0}^{y}\left[b_{22}\left(\Phi_{, x x x}^{(k)}+\bar{U}_{2, x}^{(k)}\right)+b_{12} \bar{U}_{1, x}^{(k)}\right] d y\right. \\
& -(k+1)\left[\left(\frac{b_{12} a_{44}+b_{22} a_{55}-b_{26} a_{45}}{a_{0}}-\frac{a_{23}}{a_{33}}\right) \int_{, 0}^{y} H_{, x}^{(k+1)} d y\right. \\
& -\left(\frac{b_{16} a_{44}+b_{26} a_{55}-b_{66} a_{45}}{a_{0}}-\frac{a_{36}}{a_{33}}\right) H^{(k+1)} \\
& +b_{12}\left[2 \Phi_{, x y}^{(k)}-\Phi_{, x y}^{(k)}(0,0)\right]-b_{16}\left[2 \Phi_{, y y}^{(k)}-\Phi_{, y y}^{(k)}(0.0)+\bar{U}_{.}^{(k)}\right] \\
& -b_{26}\left[2 \Phi_{, x x}^{(k)}-\Phi_{, x x}^{(k)}(0.0)+\bar{U}_{2}^{(k)}\right]+b_{66}\left[\Phi_{, x y}^{(k)}-\frac{1}{2} \Phi_{, x y}^{(k)}(0,0)\right] \\
& +(k+1)(k+2)\left[\frac{b_{12}}{a_{0}} \int_{0}^{y}\left(a_{44} L^{(k+2)}-a_{45} M^{(k+2)}\right) d v\right. \\
& -\frac{b_{22}}{a_{0}} \int_{d}^{y}\left(\int_{d}^{y}\left(a_{55} M^{(k+2)}-a_{45} L^{(k+2)}\right)_{, x} d y\right) d y \\
& \left.+\frac{b_{26}}{a_{0}} \int_{0}^{y}\left(a_{55} M^{(k+2)}-a_{45} L^{(k+2)}\right) d y\right] \\
& -\frac{b_{12}}{a_{0}} \int_{0}^{y}\left(a_{44} u_{k}-a_{45} v_{k}\right) d y+\frac{b_{26}}{a_{0}} \int_{0}^{y}\left(a_{55} v_{k}-a_{45} u_{k}\right) d y \\
& \left.-\frac{b_{22}}{a_{0}} \int_{0}^{y}\left(\int_{0}^{y}\left(a_{55} \bar{\nu}_{k}-a_{45} \bar{u}_{k}\right){ }_{, x} d y\right) d y\right\}_{x=0},
\end{aligned}
$$

and $M^{(k)}=\operatorname{Sym}\left(L^{(k)}\right), m^{(k)}=\operatorname{Sym}\left(l^{(k)}\right)$.

In the above, for efficient writing, we have introduced the notation

$$
\begin{aligned}
& \bar{u}_{k}=p_{k} \bar{u}^{(1)}+q_{k} \bar{u}^{(2)}-(k+1) d_{k} \bar{u}^{(3)} \\
& \bar{v}_{k}=p_{k} \bar{v}^{(1)}+q_{k} \bar{v}^{(2)}-(k+1) d_{k} \bar{v}^{(3)}
\end{aligned}
$$

\subsection{Loading constants}

The loading constants $p_{k}, q_{k}, \tau_{k}, d_{k}$ are defined for $k=0, \ldots, K$ by

$$
\begin{aligned}
& p_{k} \bar{I}_{11}=\bar{I}_{12} q_{k}+\oint_{\supset \Omega} X_{s}^{(k)}+\iint_{\Omega}\left\{X_{b}^{(k)}\right. \\
& +\frac{k+1}{a_{0}}\left[a_{44} \omega_{, r}^{(k+1)}-a_{45} \omega_{y}^{(k+1)}-(k+2)\left(a_{44} L^{(k+2)}\right.\right. \\
& \left.\left.\left.-a_{45} M^{(k+2)}\right)+d_{k+1}\left(\frac{a_{0}}{2 a_{33}} x-a_{44} \bar{u}^{(3)}+a_{45} \bar{v}^{(3)}\right)\right]\right\}
\end{aligned}
$$

$$
\begin{aligned}
& \tau_{k} D=p_{k} D_{1}+q_{k} D_{2}+\bar{q}_{\hat{C O S} 2}\left(x Y_{s}^{(k)}-y^{(k)}\right)+ \\
& \iint_{\Omega}\left\{x Y_{b}^{(k)}-y X_{h}^{(k)}+\frac{k+1}{a_{0}}\left[( a _ { 5 5 } x + a _ { 4 5 } y ) \left(\omega_{y}^{(k+1)}+\right.\right.\right. \\
& \left.(k+2) M^{(k+2)}\right)-\left(a_{45} x+a_{44} y\right)\left(\omega_{, x}^{(k+1)}+(k+2) L^{(k+2)}\right) \\
& \left.\left.+d_{k+1}\left(\left(a_{44} u^{-(3)}-a_{45} v^{-(3)}\right) y+\left(a_{45} \bar{u}^{(3)}-a_{55} \bar{v}^{(3)}\right) x\right)\right]\right\} \text {. } \\
& q_{k}=\operatorname{Sym}\left(p_{k}\right) \text {, } \\
& d_{k} \bar{I}_{33}=\oint_{\partial \Omega} Z_{s}^{(k)}+\iint_{\Omega}\left\{Z_{b}^{(k)}+\frac{k+1}{a_{33}}\left[a_{36} \Phi_{, x y}^{(k+1)}\right.\right. \\
& -a_{13}\left(\Phi_{, y y}^{(k+1)}+\bar{U}_{1}^{(k+1)}\right)-a_{23}\left(\Phi_{, k r}^{(k+1)}+\bar{U}_{2}^{(k+1)}\right) \\
& +\left(p_{k+1} y+q_{k+1} x\right) x y+(k+2)\left(\frac{a_{13}}{a_{0}} S_{x}^{(k+2)}\right. \\
& +\frac{a_{23}}{a_{0}} S_{y}^{(k+2)}+(k+1) d_{k+2} \frac{a_{45}+a_{36}}{2 a_{33}} x y \\
& \left.\left.\left.+\frac{a_{13} a_{44}+a_{23} a_{55}-a_{36} a_{45}+a_{0}}{a_{0}} H^{(k+2)}\right)\right]\right\} .
\end{aligned}
$$

where $\bar{I}_{i j}$ are defined in $/ 10 /$ by

$$
\begin{aligned}
& \bar{I}_{33}=\iint_{13}\left(\frac{1}{a_{33}}+\sigma_{z}^{(3)}\right), \\
& \left\{\bar{I}_{12}, \bar{I}_{22}\right\}=-\iint_{\Omega}\left(\frac{y}{a_{33}}-\sigma_{z}^{(2)}\right)\left\{-x, \nu_{\}},\right. \\
& \left\{\bar{I}_{11}, I_{21}\right\}=-\iint_{\Omega}\left(\frac{x}{a_{33}}-\sigma_{z}^{(1)}\right)\{-x, y\},
\end{aligned}
$$

and $D, \bar{\nu}_{1}, \bar{\nu}_{2}$ are

$$
\begin{aligned}
& D=-\iint_{\Omega} \frac{1}{a_{0}}\left[Q^{\varphi}\left(\varphi_{, y}+x\right)+P^{\varphi}\left(\varphi_{, x}-y\right)\right], \\
& D_{i}=\iint_{\Omega} \frac{1}{a_{0}}\left[y P^{\chi_{i}}-x Q^{\chi_{i}}-P^{\varphi} \chi_{i, x}-Q^{\varphi} \chi_{1, v}\right] .
\end{aligned}
$$

As shown in $/ 10 /$, the existence conditions for $\chi_{1}, \chi_{2}$ require a selection of the coordinate system origin so that

$$
\iint_{\Omega}\left\{\frac{x}{a_{33}}-\bar{\sigma}_{z}^{(1)}, \frac{y}{a_{33}}-\bar{\sigma}_{z}^{(2)}\right\}=\{0,0\} .
$$

\section{VERIFICATION OF SOLUTION HYPOTHESIS}

Equations (5) provide an exact solution that satisfies all requirements of the theory of elasticity. To carry out 
the above task, we employ the equilibrium equations, the compatibility equations and the outer surface boundary conditions. The process is broken into subsequent steps, where in general, at each one, we use all relevant relations that were found in previous steps.

(a) Equilibrium equations. Considering the stress terms of (5), and using the fact that the solutions of the auxiliary problems satisfy the equilibrium equations, one may verify that the equilibrium equations with $X_{b}$ and $Y_{h}$ are satisfied identically. From the third equilibrium equation (with $Z_{h}$ ) the terms of $F_{0}{ }^{(k)}$ in (17), are extracted as the coefficients of the "free term" $z^{k+1}$.

(b) Boundary conditions. From the outer contour condition of $X_{s}, Y_{s}$ one may deduce the terms for the derivatives $\frac{d}{d s} \Phi_{y}^{(k)} \cdot \frac{d}{d s} \Phi_{, x}^{(k)}$ over the contour $\partial \Omega$, see (11). From the third boundary condition of $Z$, the additional terms for the normal derivatives $D_{1}^{n} \omega^{(k)}$ presented in (17) are obtained as the coefficients of $z^{k+1}$.

(c) Compatibility equations. The terms of the strain components satisfy the compatibility equations due to the fact that the solutions of the auxiliary problems are consistent and inherently satisfy the compatibility equations.

\section{(d) Displacement interface continuity.}

The in-plane displacement components: Continuity for levels $k>K+2$, see $(9: a, b)$, follows from the fact that loading constants of Section 1.6 are not domain dependent (i.e., constants over the entire nonhomogeneous domain, $\Omega$ ). Displacement continuity for levels $k=K+2, K+1$ is achieved by the $z^{K+1}, z^{K+1}$ terms of $(9: a, b)$, which cancel out the discontinuity of $u$, $v$. For levels $k<K$ displacement continuity is part of the biharmonic problem, since by explicit use of $(9: a, b)$, the interface conditions of (10b) may also be written as

$$
\left\{u^{(k)}, v^{(k)}\right\}_{[i]}^{|j|}=\{0,0\} \text { on } \partial \Omega_{i j}
$$

The out-of-plane displacement component: Continuity of $w$ may be verified by examining (9c). This equation shows that the coefficients of $z^{k+1}, z^{k+2}$ and $z^{k+3}$ do not contribute any discontinuity due to the fact that the loading constants are not domain dependent. For lower levels this continuity condition is imposed as part (16d) of the Neumann problem for $\omega^{(k)}$.

(e) Single-valued conditions. The single-valuedtype conditions (14) for $\Phi^{(k)}$ and its first derivatives yield the expressions (19a-c) for the constants $q_{k}, p_{k}$, $\tau_{k}$, respectively.

(f) Existence of the longitudinal stress function. The existence condition (18) of a longitudinal stress function $\omega^{(L)}$ yields the definition (19d) of the constants $d_{k}$.

\section{APPLICATIONS}

\subsection{Homogeneous beam under constant distributed body force}

The loading constants of homogeneous beam are

$$
\begin{aligned}
& p_{k}=\frac{a_{33}}{l_{y}} \oint_{\partial \Omega} X_{s}^{(k)}+\frac{a_{33}}{l_{y}} \iint_{\Omega}\left\{X_{b}^{(k)}+\frac{k+1}{a_{0}}\left[a_{44} \omega_{, x}^{(k+1)}\right.\right. \\
& \left.-a_{45} \omega_{y}^{(k+1)}-(k+2)\left(a_{44} L^{(k+2)}-a_{45} M^{(k+2)}\right)\right] !, \\
& d_{k}=\frac{a_{33}}{S_{S 2}} \oint_{\sim_{0 c}} Z_{s}^{(k)}+\frac{a_{33}}{S_{S 2}} \iint_{-\infty 2}\left\{Z_{k}^{(k)}+\frac{k+1}{a_{33}}\left[a_{36} \Phi_{x y}^{(k+1)}\right.\right. \\
& -a_{13}\left(\Phi_{, y y}^{(k+1)}+\bar{l}_{1}^{(k+1)}\right)-a_{23}\left(\Phi_{, x x}^{(k+1)}+\bar{l}_{2}^{(k+1)}\right) \\
& +(k+2)\left(\frac{a_{33} a_{44}+c_{23} a_{55}-c_{36} a_{45}}{a_{1}}+1\right) H^{(k+2)} \\
& \left.\left.\left.+\left(p_{k+1} y+q_{k+1} x\right) x y+\frac{a_{13}}{a_{0}} \cdot s_{x}^{(k+2)}+\frac{a_{23}}{a_{1}} \cdot s_{y}^{(k+2)}\right)\right]\right\} .
\end{aligned}
$$

We derive here the longitudinal and biharmonic stress functions $\Phi, \omega$ for a homogeneous $Z$-monoclinic beam that undergoes constant body loads, i.e., $K=0$.

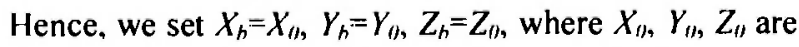
constants, while no other surface or tip loads are applied. The loading constants $p_{0}, q_{0}, \tau_{0}$ and $d_{0}$ in (22) are 
$p_{0}=\frac{S_{0}}{I_{y}} a_{33} X_{0}, q_{0}=\frac{S_{\Omega}}{I_{x}} a_{33} I_{0}, \quad \tau_{0}=0, \quad \dot{a}_{0}=a_{33} Z_{0}$

We employ (17) to find

$$
F_{0}^{\prime \prime \prime}=-a_{0} Z_{0}, \quad P_{3}=-\frac{1}{2} a_{0} Z_{0} x, \quad Q_{3}=-\frac{1}{2} a_{0} Z_{0} y .
$$

The solution $\omega$ is independent of the domain shape

$$
\omega=-\frac{Z_{0}}{4}\left(a_{55} x^{2}+2 a_{45} x y+a_{44} y^{2}\right) .
$$

For the biharmonic stress function $\Phi$ (11) show the following expansions:

$$
\begin{aligned}
& F_{0}=2 S_{\Omega}\left[\frac{X_{0}}{I_{y}}\left(a_{36} y-a_{13} x\right)+\frac{Y_{0}}{I_{x}}\left(a_{36} x-a_{23} y\right)\right], \\
& F_{i}=\left[Y_{0} y-\frac{a_{55}}{a_{0}}\left(p_{0} \chi_{1}+q_{0} \chi_{2}\right)\right] \cos (\vec{n}, y), \\
& F_{2}=\operatorname{Sym}\left(F_{\mathrm{j}}\right) .
\end{aligned}
$$

Here we used expression (8) for $H^{\prime \prime}$. and (6) for $\bar{U}_{1}, \bar{U}_{2}$.

\subsection{Homogeneous beam under linear body force distribution}

We consider here a Z-monoclinic beam of generic cross section when the body force distribution is given by $X_{b}^{(0)}=\gamma_{x} x, \gamma_{i}^{(0)}=0, Z_{b}^{(0)}=\gamma_{z} z$, while all other surface and tip loads vanish. A physical example for such a loading is the rotating beam shown in Figure 2. In such a case $\gamma_{x}=\gamma_{z}=\rho_{0} / \Omega_{0}^{2}$, where $\rho_{0}$ is the specific weight (density) of the material, and $\Omega_{0}$ is the angular velocity. We shall now discuss the two levels of this problem solution one by one.

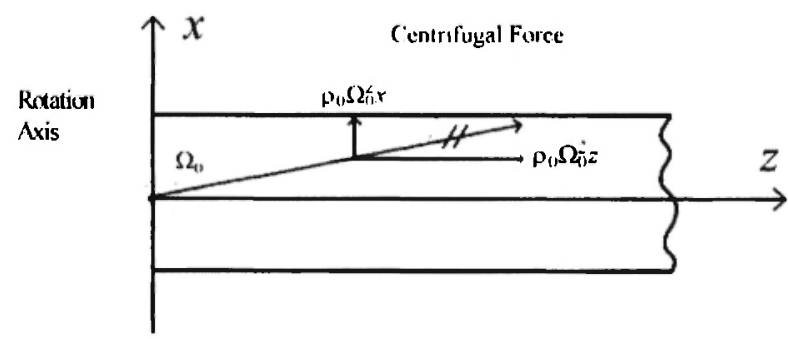

Fig. 2: Notation for rotating beam.
Level $k=1$ : Equations (19) show that

$$
p_{\mathbf{I}}=g_{\mathrm{i}}=\tau_{\mathrm{i}}-\mathbf{0}, \quad d_{\mathrm{i}}-\frac{a_{33}}{S_{\Omega}}-\int_{\Omega} \int_{b} z_{b}^{(1)}=\frac{a_{33}}{\gamma_{z}} .
$$

For the harmonic function $\omega^{(1)}$ we find

$$
\begin{aligned}
& F_{0}^{\omega^{(1)}}=-a_{0} \gamma_{z}, \\
& P_{3}^{(1)}=-\frac{1}{2} a_{0} \gamma_{z} x, \quad Q_{3}^{(1)}=-\frac{1}{2} a_{0} \gamma_{z} y .
\end{aligned}
$$

Analogously to the solution presented in (23), $\omega^{(1)}$ is independent of the domain shape

$$
\omega^{(1)}=-\frac{\gamma_{z}}{4}\left(a_{55} x^{2}+2 a_{45} x y+a_{44} y^{2}\right)
$$

Level $k=0$ : Equations (19) and Green's Theorem show that

$$
\begin{aligned}
& p_{0}=\frac{a_{33}}{l_{y}} \int_{\Omega}\left[x_{b}^{(i)}+\frac{1}{a_{0}}\left(a_{44} \omega_{x}^{(1)}-a_{45} \omega_{y}^{(1)}\right)\right]=0, \\
& q_{0}=\operatorname{Sym}\left(p_{0}\right)=0
\end{aligned}
$$

and that $d_{0}=0, \tau_{0} \neq 0$. Equations (17) show that $\omega^{(0)}=0$. At this stage one should solve the biharmonic problem (10) for $k=0$.

\subsection{Non-homogeneous beam under constant axial body force}

Consider a non-homogeneous Z-monoclinic beam that undergoes constant body force in the $z$-direction, $Z_{h}=Z_{b}=$ const., $\left(X_{h}=\gamma_{h}=0\right)$, while no other surface or tip loads are applied. The loading constants of (19) are $p_{0}=q_{0}=0, d_{0}=Z_{0} S_{\Omega} / \bar{I}_{33}$, and

$$
\begin{gathered}
\tau_{0}=\frac{\check{\Sigma}_{0} \tilde{j}_{\Omega}}{D \bar{I}_{33}} \iint_{\Omega} \frac{1}{a_{0}}\left[\left(a_{44} \bar{u}^{(3)}-a_{45} \bar{v}^{(3)}\right) y\right. \\
\left.-\left(a_{55} \bar{v}^{(3)}-a_{45} \bar{u}^{(3)}\right) x\right]
\end{gathered}
$$

where $D, \bar{l}_{33}$ are given by (20:a), (21:a). Since $K=0$, only the stress functions $\Phi, \omega$ and the auxiliary functions $L, M$ of level $k=0$ should be considered, and 
hence, for the sake of convenience, in what follows we shall omit the index superscript. The Neumann problem (16) for $\omega(x, y)$ should be written with

$$
\begin{aligned}
& P^{\omega}=d_{0}\left(-\frac{a_{0}}{2 a_{33}} x+a_{44} \bar{u}^{(3)}-a_{45} \bar{v}^{(3)}\right), \\
& Q^{(\nu}=\operatorname{Sym}\left(P^{\omega}\right) \\
& F_{0}^{(j)}= d_{0}\left(a_{44} \bar{\varepsilon}_{x}^{(3)}+a_{55} \bar{c}_{y}^{(3)}-a_{45} \bar{\gamma}_{y}^{(3)}+a_{0} \bar{\sigma}_{z}^{(3)}\right)-a_{0} Z_{0} . \\
& F^{(\omega, y}=-\hat{u}_{\bar{v}}\left\{\frac { 1 } { 4 a _ { 3 3 } } \left[\left(2 a_{13}+a_{55}\right) x^{2}\right.\right. \\
&\left.\left.\quad+2\left(a_{36}+a_{45}\right) x y+\left(2 a_{23}+a_{44}\right) y^{2}\right]\right\}_{[i]}^{[j]},
\end{aligned}
$$

while for convenience we assume $\omega(0,0)=0$. The biharmonic problem for $\Phi(x, y)$ should have the type of (10) with $F_{0}-F_{1}=F_{2}=0$ and

$$
L=A+B \tau_{0} . \quad M=\operatorname{Sym}(L)
$$

where $B$ depends on $\varphi$ and $A$ is a linear differential operator of $\Phi$.

The stresses become

$$
\begin{aligned}
\sigma_{x} & =\Phi_{. w}-z d_{0} \bar{\sigma}_{x}^{(3)}, \quad \sigma_{y}=\operatorname{Sym}\left(\sigma_{x}\right) \\
\tau_{x y} & =-\Phi_{. x y}-z d_{0} \bar{\tau}_{x y}^{(3)}, \\
\sigma_{z} & =\frac{1}{a_{33}}\left(a_{36} \Phi_{. y y}-a_{13} \Phi_{y y}-a_{23} \Phi_{x x}\right)-\left(\frac{1}{a_{33}}+\sigma_{z}^{(3)}\right) z d_{0}, \\
\tau_{v z} & =\frac{1}{a_{0}}\left(a_{55} \omega_{. y}-a_{45} \omega_{x}\right)-\frac{d_{0}}{a_{0}}\left(a_{55} v^{(3)}-a_{45} \bar{u}^{(3)}\right) \\
& =\frac{z \tau_{0}}{a_{0}}\left(a_{55} \varphi_{. y}-a_{45} \varphi_{. x}-Q^{\varphi}\right)+\frac{d_{0} y}{2 a_{33}}, \\
\tau_{x z} & =\operatorname{Sym}\left(\tau_{y z}\right) .
\end{aligned}
$$

Once $\Phi, \omega$ are determined, one needs to superimpose suitable St. Venant's solutions of $/ 10 /$ in order to cancel out the tip resultants which are induced by the above stresses.

Let a beam cross-section be geometrically symmetric about the $x$-axis with anti-symmetric lamination, based on orthotropic material turned about angles $\pm O$, for example, a non-homogeneous rectangle. see Figure 3.

Recall that elastic moduli are identical in two domains $\Omega_{111}, \Omega_{|2|}$ except for $a_{16}, a_{26}, a_{36}$ and $a_{45}$ that are of identical magnitude but opposite signs. The same is true for reduced elastic constants $b_{u}$.

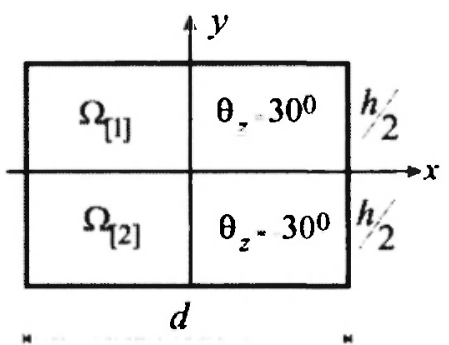

Fig. 3: A $y$-laminated rectangle, $N=2$.

By (initially) placing the coordinate system at the cross-section midpoint, we show that the solution for the third auxiliary problem, see $/ 10 /$, is zero $\Phi^{(3)}=0$, and $\bar{\varepsilon}_{y}^{(3)}=F_{y}^{(3)}=\bar{\pi}_{z}^{(3)}=0$. Hence $\bar{l}_{33}=S_{\Omega 2} / a_{33}$ and the displacements are rigid,

$$
\bar{u}^{(3)}=\frac{a_{36}}{2 a_{33}} y . \quad \bar{v}^{(3)}=-\frac{a_{36}}{2 a_{33}} x .
$$

The loading constants are $p_{0}=q_{0}=0, d_{0}=Z_{0} a_{33}$,

$$
\tau_{0}=\frac{\vec{z}_{0}}{2 D a_{0}} \iint_{\Omega} a_{36}\left(a_{44} v^{2}+a_{55} x^{2}\right)=0 .
$$

Since in this case the biharmonic problem $(10)$ is homogeneous, $\Phi=0$, we obtain

$$
\omega=-\frac{Z_{0}}{4}\left(a_{55} x^{2}+2 a_{45} x y+a_{44} y^{2}\right)+\frac{Z_{0}}{2} a_{36}^{[1]} \bar{\varphi}
$$

where $\bar{\varphi}$ is known harmonic function with the symmetry $\bar{\varphi}(x,-y)=\bar{\varphi}(x, y)$, see Figure 4 .

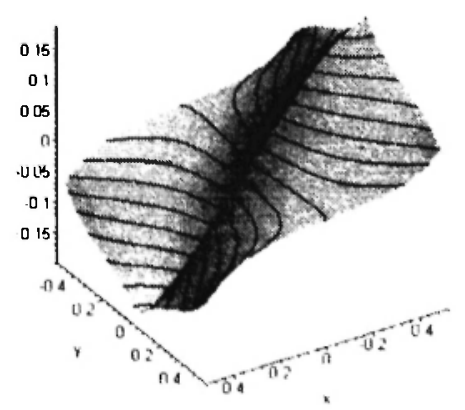

Fig. 4: The function $\bar{\varphi}$. 
The stress solution that does not produce tip loads, i.e., see (4),

$$
\iint_{\Omega_{\text {tip }}}\left\{\sigma_{z}, \sigma_{z} y, \sigma_{z} x, \tau_{x z}, \tau_{y z}, \tau_{y z} x-\tau_{x z} y\right\}=\{0,0,0,0,0,0\}
$$

is

$$
\begin{aligned}
& \sigma_{x}=\sigma_{y}=\tau_{x y}=0, \sigma_{z}=Z_{0}(z-l), \\
& \tau_{y z}=\frac{Z_{0} a_{2 !}^{[1]}}{2 c_{0}}\left[a_{55} \bar{\varphi}_{, y}-a_{45} \bar{\varphi}_{, x}+\frac{a_{2 \varsigma}}{a_{36}^{[1 !}}\left(a_{55} x+a_{45} y\right)\right], \\
& \tau_{x z}=\operatorname{Sym}\left(\tau_{y z}\right) .
\end{aligned}
$$

Referring to (9) the axis extension becomes

$$
u(0,0, z)=v(0,0, z)=0, w(0,0, z)=Z_{0} a_{33} z(l-z / 2) .
$$

The shear stresses $\tau_{y z}$ and $\tau_{x z}$ for $Z_{0}=1$ are presented in Figures 5.
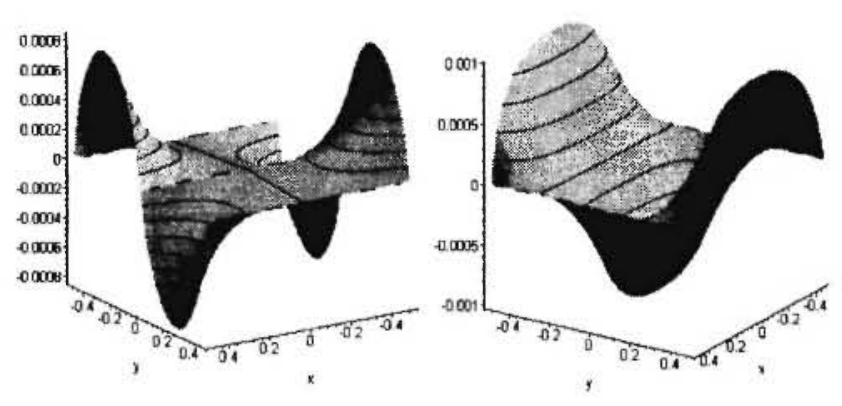

(a) $\tau_{y z}(x, y)$.

(b) $\tau_{x z}(x, y)$.

Fig. 5: The shear stresses.

\section{REFERENCES}

1. J.H. Michell, The theory of uniformly loaded beams. Journal of Mathematics, 32, 28-42 (1901).
2. E. Almansi, Sopra la deformazione dei cilindri sollecitati lateralmente. Atti della Academia Nazionale dei Lincei Rendiconti, 1901, v. 10, Nota I: 333-338, Nota II: 400-408.

3. G.Y. Dzhanelidze, The Almansi problem. Proceedings of Leningrad Polytechnic Institute, 210, 25-38 (1960) (in Russian).

4. A.E.H. Love, $A$ treatise on the mathematical theory of elasticity. Fourth edn. New York, NY, USA: Dover Publications, Inc., 1927

5. N.I. Muskhelishvili, Some Basic Problems of the Mathematical Theory of Elasticity. P. Noordhoof Ltd, Groningen, Holand, 1953.

6. A.S. Kosmodamianskii, Bending of anisotropic beam under generic distributed load. Ingenernii Sbornik, 24, 114-126 (1956) (in Russian).

7. A.K. Ruchadze, On one problem of elastic equilibrium of homogeneous isotropic prismatic bar. Trudii Gruzinskogo Politechnicheskogo Instituta, 3 (176) 208-218 (1975) (in Russian).

8. A.K. Ruchadze and R.A. Berekashvili, On one general problem of Almansi. Soobsheniya Akademii Nauk of G.S.S.R, 100 (3), 561-564 (1980) (in Russian).

9. R.T. Zivsivadse and R.A. Berekashvili, Generalization of Almansi problem for compound anisotropic cylindrical beams. Georgian Polytechnical Institute, 9 (279), 130-135 (1984) (in Russian).

10. M. Kazar (Kezerashvili), V. Rovenski, $M$. Grebshtein and O. Rand, Non-Homogeneous Uncoupled Beam under Tip Loads. To be published.

11. V.V. Novozhilov, Theory of Elasticity. Pergamon Press, Ltd., 1961.

12. S.G. Lekhnitskii, Theory of Elasticity of an Anisotropic Elastic Body. Mir Publishers, Moscow, 1981. 